ECONOMÍA: TEORÍA Y PRÁCTICA • Nueva Época, número 44, enero-junio 2016,

pp. 51-82, http://www.izt.uam.mx/economiatyp/ojs

\title{
Revisión de la hipótesis de convergencia mediante cointegración en panel: el caso de América Latina*
}

\section{Review of the Convergence Hypothesis by means of Panel Cointegration: Latin America's Case}

\author{
Domingo Rodríguez Benavides, ** Francisco López-Herrera*** \\ y Miguel Ángel Mendoza González****
}

\section{RESUMEN}

Este trabajo reexamina la hipótesis de la convergencia del PIB per cápita en una muestra de países de América Latina con respecto a dos referentes: el promedio de la región y el de Estados Unidos. A diferencia de Rodríguez, Perrotini y Venegas (2012) y de Rodríguez, Perrotini y Mendoza (2014), se emplean pruebas de raíces unitarias y de estacionariedad, así como de cointegración en panel con rupturas estructurales. Los resultados de éstas no corroboran la hipótesis de convergencia que brindan las de raíces unitarias y de estacionariedad en panel con rupturas aplicadas a la versión restricta, lo que se confirma con las estimaciones del parámetro de convergencia en la versión irrestricta de la prueba.

Clasificación JEL: C23, O47, O54.

Palabras clave: convergencia, raíces unitarias en panel, cointegración en panel, paneles cointegrados.

\begin{abstract}
The Latin American countries' PIB per capita convergence hypothesis is reviewed considering two proxies: the region's average and the USA economy. Unlike Rodríguez, Perrotini and Venegas (2012), or Perrotini and Mendoza (2014), tests of unit roots, stationarity and panel cointegration with structural breaks are used. When applied to the restricted version, the tests of panel cointegration do not support the evidence provided by the panel unit roots and stationarity tests stating convergence, although the breaks are taken into account. The convergence parameter estimation also casts out similar results in the case of the irrestricted test.

JEL classification: $\mathrm{C} 23, \mathrm{O} 47, \mathrm{O} 54$.

Keywords: convergence, panel unit roots, panel cointegration, cointegrated panels.

* Fecha de recepción: 27/02/2014. Fecha de aprobación: 11/02/2016. Los autores agradecen las observaciones y recomendaciones efectuadas por los dictaminadores anónimos al presente trabajo.

** Departamento de Sistemas de la Universidad Autónoma Metropolitana-Azcapotzalco. Correo electrónico: dorobe@correo.azc.uam.mx.

*** División de Investigación de la Facultad de Contaduría y Administración de la Universidad Autónoma de México. Correo electrónico: francisco_lopez_herrera@yahoo.com.mx.

**** División de Estudios de Posgrado de la Facultad de Economía de la Universidad Nacional Autónoma de México. Correo electrónico: mendozag@unam.mx.
\end{abstract}




\section{INTRODUCCIÓN}

Los diferenciales en el crecimiento económico han sido objeto de reciente y recurrente escrutinio desde muy diversos enfoques (Carrion-i-Silvestre y GermanSoto, 2007; Mankiw, Romer y Weil, 1992). La mayoría de los estudios acerca de la convergencia económica se han concentrado en las naciones desarrolladas prestando atención en menor medida a los países en desarrollo. No obstante, la investigación sobre la convergencia en éstos ha crecido considerablemente en los últimos años.

La evolución de la actividad económica de largo plazo en América Latina permite identificar momentos históricos en que las diferencias económicas entre los países tienden a reducirse, así como otros en que se amplían cada vez más. Estas aproximaciones y distanciamientos se identifican como procesos de convergencia y divergencia, y los momentos de cambio de la convergencia a la divergencia o viceversa se caracterizan como cambios estructurales.

Se retoman los nuevos desarrollos analíticos de teoría económica regional, con base en los enfoques neoclásicos, keynesianos y de la nueva geografía económica, para desarrollar argumentos que muestren que la dicotomía convergencia-divergencia se está modificando y explicar la coexistencia de los dos procesos (Rodríguez, Mendoza y Perrotini, 2015). A su vez, desde el punto de vista empírico, la estrategia que se propone consiste en enlazar los conceptos de convergencia y divergencia económica con la metodología econométrica de pruebas de hipótesis de raíces unitarias y/o estacionariedad en panel, para comprobar si los PIB per cápita en cada región siguen un proceso estacionario (convergente) o de camino aleatorio (divergente). Pero si el supuesto es que las regiones presentan cambios en las relaciones económicas de largo plazo y provocan con ello que los procesos estructurales de convergencia-divergencia se modifiquen, entonces se propone utilizar la metodología econométrica de pruebas de hipótesis de raíces unitarias y/o estacionariedad en panel con cambio estructural.

En este trabajo se analiza la hipótesis de convergencia regional en América Latina, considerando que los procesos de convergencia y de divergencia regional beta pueden ser identificados en un proceso de crecimiento económico con cambio estructural, para lo cual se utilizan pruebas de raíces unitarias y de estacionariedad, así como de cointegración en panel, con y sin rupturas estructurales. El trabajo está compuesto de la siguiente manera: la sección I brinda una breve revisión de la literatura empírica sobre la convergencia en América Latina, así como de la especificación de las pruebas de convergencia absoluta y condi- 
cional. En la segunda, se muestran la metodología econométrica empleada y los datos, mientras que en la tercera se brindan los resultados de las pruebas econométricas. Por último, se enuncian las conclusiones.

\section{REVISIÓN DE LA LITERATURA}

\section{Sobre la convergencia en América Latina}

Mendoza (2007) señala que entre 1950 y 2000 se duplicó el promedio regional del ingreso real per cápita en América Latina, destacando Argentina, Uruguay, Venezuela y México por sus mayores niveles entre 1950 y 1975; no obstante, con base en su análisis, dicho autor sostiene que el proceso de convergencia en la región latinoamericana no ha sido ininterrumpido y que la transformación del modelo económico ha influido en forma definitiva. El cambio del patrón de crecimiento económico ha hecho que el subperiodo 1980-2000 se caracterice por un proceso de divergencia, en el cual se observa un notorio contraste entre países con un ritmo lento (Nicaragua, Costa Rica y El Salvador) y otros con altos niveles de crecimiento, como Chile.

Álvarez, Lucas y Delgado (2009) estudian la globalización como factor condicionante del proceso de convergencia entre 16 países de América Latina. Su análisis abarca el periodo 1970-2005 con base en técnicas econométricas para panel de datos y por medio de los indicadores de convergencia sigma y convergencia beta. Encuentran que la perspectiva económica y social del proceso de globalización ha acelerado el proceso de convergencia económica, respecto al ingreso per cápita, pero la globalización política no muestra impactos significativos. Además, que en algunos casos los países con menor renta tienen una tasa de crecimiento superior que los de mayor renta (convergencia beta), por ejemplo, República Dominicana en comparación con Venezuela, sin embargo, también observan lo opuesto: tasas de crecimiento más grandes en países con mayor nivel de renta. Un caso encontrado durante el periodo de su análisis es el de Trinidad y Tobago, que había crecido a mayores tasas que Bolivia. También hallan que el indicador de integración económica tiene un efecto positivo y significativo en la disminución de las disparidades en el ingreso, así como una influencia favorable en la velocidad del proceso de convergencia.

En su análisis sobre la convergencia entre los países latinoamericanos, Martín (2010) estudia, mediante la técnica de datos de panel dinámico, la evolución de las disparidades en el nivel del ingreso de esas naciones entre 1950 y 
2008. Al igual que Fajnzylber (1990), Martín observa que en el horizonte de su análisis ha aumentado la dispersión en el ingreso per cápita como consecuencia de las disparidades en la actividad económica, no obstante que los PIB per cápita de los países latinoamericanos se han acercado al promedio regional entre $1950 \mathrm{y}$ 1980. El país con más ingreso per cápita es Chile (1.67 veces por arriba de dicho promedio), seguido por Argentina (1.6) y Uruguay (1.46). Panamá y República Dominicana han mejorado notablemente a partir de la década de los noventa, superando ya ese promedio. Los países con peor desempeño son Honduras, Bolivia, Nicaragua y Guatemala, con un ingreso per cápita menor a $50 \%$ del promedio de referencia. México, a pesar del Tratado de Libre Comercio de América del Norte (TLCAN) y la fuerte concentración de sus exportaciones a Estados Unidos (EU), apenas oscila en torno al promedio durante todo el periodo considerado. De acuerdo con los resultados del modelo econométrico usado por Martín (2010), el proceso de convergencia del ingreso per cápita de los países de la región ha sido lento hasta 1985, año a partir del cual se vuelve dinámico, lo cual, junto con las disparidades observadas, hace concluir a este autor que el proceso de convergencia beta es condicional, por grupos de países, hacia estados diferenciados, los cuales dependen positivamente de la tasa de ahorro-inversión y negativamente del gasto público.

Sanguinetti y Villar (2012) destacan que en la región latinoamericana, más que de convergencia, se puede hablar de divergencia, pues sus países pueden caracterizarse como un caso típico del fenómeno llamado "la trampa del ingreso medio", la cual Zheng (2011) define como la situación de estancamiento relativo a la que han llegado las economías de muchos países de la región debido a los costos salariales, que les impiden competir en los mercados internacionales, y a las severas dificultades que padecen para competir con productos intensivos en conocimiento y tecnología.

En su análisis, estos investigadores muestran que a principios del siglo pasado el ingreso per cápita en Argentina, Uruguay y Chile era superior a 50\% del de EU. Sin embargo, después de que en los años treinta en Argentina y Uruguay esa proporción alcanzara máximos en torno a $75 \%$ y $60 \%$, respectivamente, en ambos casos se ha ubicado alrededor de $30 \%$ en los primeros años del presente siglo. El caso de Chile es diferente, pues aunque a principios del siglo xx su ingreso per cápita era de $50 \%$ del de EU, cayó a $24 \%$ a mediados de los años ochenta. Sin embargo, después de esa reducción se recuperó hasta llegar a $42 \%$ en 2008, permitiéndole ubicarse como país más rico de la región. El ingreso per cápita en Venezuela, comparado también con el de EU, fue de $20 \%$ durante las 
dos primeras décadas del siglo pasado, creciendo desde esa época en forma muy fuerte y sostenida gracias al boom petrolero hasta llegar a niveles superiores a $80 \%$ en los años cincuenta. Después de este vigoroso crecimiento, el indicador en Venezuela declinó de forma también constante hasta ubicarse en torno a 30\% en años recientes. Tomando en cuenta todo ese largo periodo, se puede decir que el ingreso per cápita en Brasil ha tenido una mejoría marginal, pasando de representar $17 \%$ del de eU a principios del siglo xx a un poco más de $20 \%$ en los últimos años. Sanguinetti y Villar encuentran que, al igual que en el caso brasileño, México tuvo una tendencia hacia la convergencia entre los años cuarenta y setenta, sin embargo, señalan que no se ha observado un proceso de convergencia en el largo plazo, pues el ingreso per cápita en México es actualmente de alrededor de $25 \%$ del de EU, siendo ligeramente superior a $30 \%$ en los inicios del siglo pasado. A principios de esa centuria, Colombia y Perú tenían ingresos relativos muy bajos, pero aumentaron hasta alcanzar un nivel cercano a $30 \%$ en los años treinta, revirtiéndose posteriormente. En Colombia se mantuvo estable en torno a $20 \%$ a partir de los años cincuenta, en tanto que en Perú volvió a aumentar hasta un nivel de casi $30 \%$ alrededor de 1960 , pero cayendo desde entonces a los niveles inferiores a $20 \%$ que se observan hasta la actualidad.

Evans (1997) demuestra que cuando se incorporan variables de control en las especificaciones iniciales para probar convergencia, a pesar de que éstas controlan $90 \%$ de la varianza de los niveles de PIB per cápita en el estado estacionario, el límite de probabilidad del estimador de mínimos cuadrados del coeficiente en el ingreso inicial (que es el indicador de convergencia) es aproximadamente igual a la mitad de su verdadero valor. Por dicha razón, no es conveniente realizar inferencias empleando este tipo de regresiones.

Dentro de los estudios que han empleado técnicas de series temporales, destacan Linden (2000), quien estudia el conjunto de países de la Organización para la Cooperación y el Desarrollo Económicos (OCDE) aplicando las pruebas de raíces unitarias de Dickey Fuller aumentada (ADF) y de Kwiatkowski, Phillips, Smichdt y Shin (KPSS) por pares, encontrando convergencia sólo para Noruega, Suecia y Reino Unido. Por otra parte, Amable y Juillard (2000), aplicando las mismas pruebas en una muestra de 53 países, encontraron que la prueba ADF casi nunca aceptó convergencia, con excepción de Dinamarca y Alemania.

Asimismo, Camerero, Flores y Tamarit (2002), al estudiar los países que conforman el Mercosur, a través de una prueba ADF por pares y de modelos panel, encuentran evidencia de convergencia entre algunos de ellos. Por su parte, Easterly, Fiess y Lederman (2003) analizan la hipótesis de convergencia entre 
México y EU con la prueba de Johansen, encontrando evidencia de convergencia condicional. Por último, Cheung y García (2004) analizan el caso del grupo de los siete (G-7) por medio de una prueba ADF por pares y de estudios de panel; su estudio señala que la primera no muestra evidencia de convergencia.

Cermeño y Llamosas (2007) emplean las versiones restricta e irrestricta para probar la hipótesis de convergencia del PIB per cápita de seis países emergentes con respecto a EU. Con tal fin, realizan pruebas de cointegración bajo posible cambio estructural, siguiendo el enfoque de Gregory y Hansen (1996). Sus resultados sugieren que en la mayoría de los casos no se encuentra evidencia a favor de la convergencia en presencia de cambio estructural y que las brechas de ingreso per cápita de los países considerados con respecto de EU son consistentes con procesos de no convergencia.

Rodríguez, Perrotini y Venegas-Martínez (2012) examinan la hipótesis de convergencia en una muestra de 17 países de América Latina con respecto a EU en el periodo 1970-2010. A través de pruebas de raíces unitarias y de cointegración en panel sin rupturas no encuentran evidencia de convergencia absoluta, pero sí de convergencia condicional.

Por otra parte, Rodríguez, López-Herrera y Venegas-Martínez (2014), a través de un modelo no lineal de coeficientes variantes de un solo factor, encuentran evidencia de convergencia relativa en cuatro grupos o clubes de países, con las series del PIB per cápita sin filtrar, pero cuando la prueba se efectúa con las series procesadas a través del filtro de Hodrick-Prescott, no detectan presencia de convergencia, lo cual los lleva a concluir que hay grupos de países de la región que se encuentran sujetos a impactos externos comunes más que a un proceso de convergencia entre ellos.

De igual forma, Rodríguez, Perrotini y Mendoza (2014) -a través de un análisis por periodos de una muestra de países de América Latina efectuado con pruebas de raíces unitarias en panel de segunda generación que toman en cuenta la posibilidad de dependencia de sección cruzada en paneles heterogéneos y a través de los estimadores del grupo de medias (mean group) y el estimador de medias agrupadas (pooled mean group)- encuentran evidencia mixta de convergencia económica en el periodo 1950-1990 y de convergencia condicional hacia el promedio de la región y con respecto a eU de 1990 a 2010.

Adicionalmente, Rodríguez, Mendoza y Perrotini (2015) analizan la hipótesis de convergencia mediante un modelo panel TAR (threshold autoregressive) de crecimiento no lineal, propuesto inicialmente por Beyaert y Camacho (2008), para el periodo 1950-2010. Esta metodología combina tres enfoques: el 
modelo de umbral, las pruebas de raíces unitarias en panel y el cálculo de valores críticos a través de bootstrapping. Rodríguez, Mendoza y Perrotini (2015) dividen su análisis en dos grupos de países de acuerdo con su riqueza. Sus resultados sugieren que el modelo lineal es superior al no lineal y no encuentran evidencia de convergencia, parcial o absoluta en virtud de que no logran identificar un grupo de naciones con mayores niveles de ingreso por habitante que se comporte como la economía de referencia.

\section{Especificación de la pruebas de convergencia absoluta y condicional}

Uno de los conceptos de convergencia comúnmente empleados es el de $\beta$-convergencia. Se dice que existe $\beta$-convergencia entre países o regiones si hay una relación negativa entre la tasa de crecimiento del ingreso per cápita y el valor inicial de éste, lo cual implica que las economías más pobres crecen a un ritmo más acelerado que las ricas. En la década de los noventa, diversos estudios se enfocaron en la relación entre la tasa de crecimiento del ingreso per cápita y diferentes medidas de estándares de vida en secciones cruzadas para investigar el proceso de crecimiento. Esos estudios se enfocaron en un modelo de la forma:

$$
g_{i}=\alpha X_{i}+\beta y_{i 0}+\varepsilon_{i}
$$

donde $g_{i}$ es la tasa de crecimiento por país; $y_{i 0}$, el valor de la variable a nivel nacional en el comienzo del periodo de análisis; $X_{i}$, variables por país para controlar efectos específicos de cada uno de ellos, y $\varepsilon_{i}$, el término de perturbación. El valor inicial de la variable, $y_{i 0}$, se incluye con la finalidad de probar la hipótesis de convergencia (Durlauf, 2000). De esta forma, si el valor de $\beta$ resulta negativo en (1), entonces existe $\beta$-convergencia. En términos de la ecuación (1), una forma de probar la versión absoluta, o incondicional, de la convergencia consiste en excluir las variables de control específicas de cada país y verificar que el signo de $\beta$ sea negativo, mientras que una prueba de convergencia condicional se lleva a cabo incorporando las variables de control (Barro y Sala-i-Martin, 2004). Sin embargo, distintos estudios critican esta forma de probar la convergencia. Por ejemplo, Bernard y Durlauf (1996) afirman que una vez que este análisis se aplica a un conjunto de datos de países a través de un modelo correctamente especificado con múltiples estados estacionarios, entonces un coeficiente $\beta$ negativo para toda la muestra puede atribuirse a una submuestra de esos países que converjan hacia el grupo específico de estados estacionarios. Adicionalmente, Quah 
$(1993,1996)$ sugiere que estas pruebas de la hipótesis de convergencia incurren en la falacia de Galton, es decir, que una vez que las tasas de crecimiento son devueltas a los niveles iniciales, un coeficiente $\beta$ negativo se debe a una regresión a la media, lo cual no necesariamente implica convergencia.

La gran mayoría de estudios que han utilizado (1) han tendido a ignorar los patrones subyacentes de heterogeneidad en los datos al aplicar un modelo de regresión idéntico para todos los países de la muestra. Algunos de ellos usan variables dummy para América Latina o para el África subsahariana con el fin de controlar las diferencias en el proceso de crecimiento en esos grupos de países; sin embargo, esto no es suficiente para capturar los estadísticos de los grupos en el conjunto de datos. Al respecto, Bernard y Durlauf (1995) evalúan la posibilidad de convergencia utilizando el siguiente modelo:

$$
y_{i t}=\alpha_{i j}+\beta y_{j t}+\varepsilon_{i j t},
$$

donde $y_{i t}$ es el ingreso por persona del país a estudiar; $y_{j i}$, el ingreso por persona del país líder o de referencia, y $\alpha_{i j}$, una constante que denota diferencias permanentes entre las dos economías (Cermeño y Llamosas, 2007). Si hay convergencia, las diferencias entre dos economías tenderán a reducirse a través del tiempo, es decir, se requiere que $\alpha_{i j}=0$ para que las diferencias se hayan eliminado por completo (convergencia absoluta). De no cumplirse lo anterior, se tenderá a un determinado nivel diferenciado (convergencia condicional). De esta manera, el cumplimiento de la hipótesis de convergencia absoluta requiere que $\beta=1 \mathrm{y}$ $\alpha_{i j}=0$. Si $\alpha_{i j} \neq 0$, entonces hay evidencia de convergencia condicional.

Una forma alternativa y relativamente sencilla de probar la convergencia absoluta consiste en verificar el orden de integración de la diferencia entre el ingreso por persona del país a estudiar y el del país líder o de referencia; ambos, en logaritmos naturales:

$$
y_{i t}-y_{j t}=\varepsilon_{t} .
$$

A esta versión de la prueba se le conoce como la versión restricta. De acuerdo con Cheung y Garcia (2004), si se prueba la hipótesis nula de raíz unitaria en la serie resultante en (3), los resultados pueden sesgarse hacía la aceptación de la hipótesis de no convergencia debido al reducido poder de las pruebas de raíces unitarias, por lo que ellos proponen evaluar la hipótesis de convergencia a través de pruebas cuya hipótesis nula sea la estacionariedad. En caso de que para 
ambas pruebas, que tienen como hipótesis nula la raíz unitaria y la estacionariedad, no sea posible rechazar tales hipótesis al mismo tiempo, entonces los datos no pueden proveer evidencia para aceptar o rechazar la hipótesis de convergencia.

Por lo que respecta a la versión irrestricta de la prueba, ésta no se supone a priori y se emplea el modelo (2) para estimar los parámetros $\alpha_{i j} \mathrm{y} \quad \beta$. De este modo, la hipótesis de no convergencia se evalúa aplicando la prueba de raíz unitaria a los errores estimados en este modelo. Con este enfoque, la hipótesis nula establece que no hay cointegración entre los ingresos por persona del país considerado, con respecto de la economía de referencia. Además, esta versión de la prueba tiene la ventaja de que es posible determinar si la constante es significativa $\mathrm{y}$, por lo tanto, puede mostrar evidencia de convergencia condicional, así como verificar si el vector $(1,-1)$ del modelo restricto se cumple o no.

La prueba planteada en (3) para probar la hipótesis de convergencia entre dos países se puede extender para un modelo en panel que comprenda un conjunto de países, de la siguiente forma:

$$
D_{1} y_{i t}=y_{i t}-y_{t t},
$$

donde $y_{i t}$ es el ingreso per cápita del país $i$ en el momento $t, \mathrm{y} y_{t t}$ es el ingreso per cápita del país de referencia en el tiempo $t$, ambos en logaritmos. En consecuencia, la hipótesis de convergencia entre dos economías se puede probar por medio del análisis de integración y cointegración en panel cuando los ingresos por habitante de ambas regiones no son estacionarios (Díaz-Pedroza, SánchezVargas y Mendoza-González, 2009), lo cual se puede llevar a cabo aplicando distintas pruebas de raíces unitarias en panel al conjunto de series resultante de (3).

\section{METODOlOGíA ECONOMÉTRICA Y DATOS}

\section{Pruebas de raíces unitarias en panel con y sin rupturas}

\section{a) La prueba de Schmidt y Phillips (1992) sin rupturas}

Im, Lee y Tieslau (2005) consideran el siguiente modelo con el fin de probar estacionariedad en una serie de datos en panel:

$$
y_{i t}=\delta_{i}^{\prime} z_{i t}+e_{i t}
$$




$$
e_{i t}=\phi_{i} e_{i, t-1}+\varepsilon_{i, t}
$$

donde $y_{i, t}$ se refiere a la variable $y$ para la unidad $i$ en el tiempo $t ; z_{i t}$ es un conjunto de variables exógenas con vector de parámetros $\delta_{i}$, y $e_{i t}$ es el término de perturbación. Si $z_{i t}=[1, t]^{\prime}$ entonces el PIB es el mismo que la prueba sin rupturas de Schmidt y Phillips (1992). En este contexto, la hipótesis nula de raíz unitaria se especifica como $\phi_{i}=1$ en (6).

\section{b) La prueba de Im, Lee y Tieslau (2005) con una y dos rupturas}

En el caso de una ruptura estructural, el vector $z_{i t}$ toma la forma $\left[1, t, D_{i t}, D T_{i t}\right]^{\prime}$ en la ecuación (5). En el caso de que una ruptura estructural ocurra para la serie $i$ en la fecha $T B^{i}$, entonces la variable dummy $D_{i t}=1$ si $t>T B^{i}$, y 0 de otra forma; y $D T_{i t}=t-T B^{i}$ si $t>T B^{i}$, y 0 de otra forma. De una manera similar, esta prueba se puede extender a dos rupturas estructurales si $z_{i t}$ se especifica cómo $\left[1, t, D_{1, i t}, D_{2, i t}, D T_{1, i t}, D T_{2, i t}\right]^{\prime}$. Si la primera y segunda rupturas ocurren en los puntos $T B_{1}^{i}$ y $T B_{2}^{i}$, respectivamente. Entonces, para $j=1,2, D_{j, i t}=1 \mathrm{si} t>T B_{j}^{i}$, y 0 de otra forma; y $D T_{j, i t}=t-T B_{j}^{i}$ si $t>T B_{j}^{i}$ y 0 de otra forma.

El primer paso en el procedimiento de la prueba de Im, Lee y Tieslau (ILT) consiste en estimar la siguiente ecuación de regresión:

$$
\Delta y_{i t}=\delta_{i} \Delta z_{i t}+\beta_{i} \tilde{s}_{i, t-1}+\sum_{j=1}^{p_{i}} p_{i j} \Delta \tilde{s}_{i, t-j}+\varepsilon_{i, t},
$$

donde $\Delta y_{i t}$ y $\Delta z_{i t}$ son los valores en primeras diferencias de $y_{i t}$ y $z_{i t}$, respectivamente; $\tilde{s}_{i, t-1}$, el valor sin tendencia de $y_{i t}$ con el correspondiente coeficiente $\beta_{i}=1-\phi_{i}$, y $\Delta \tilde{s}_{i, t-j}$, el término añadido para corregir la posible presencia de corrección serial, como es sugerido por Amsler y Lee (1995). La presencia de una raíz unitaria en $y_{i t}$ para el estado $i$ implica que $\beta_{i}=0$. Entonces, el estadístico de prueba de multiplicadores de Lagrange (LM) univariado para la serie temporal $i$ puede calcularse a partir de la ecuación (7) para $\beta_{i}=0$ y el estadístico resultante es denotado por $L M_{i}^{T}$.

El segundo paso es calcular el estadístico de prueba LM promediando los estadísticos de prueba de raíz unitaria LM univariados:

$$
\overline{L M}_{N T}=\frac{1}{N} \sum_{i=1}^{N} L M_{i}^{T}
$$


La normalización provee el siguiente resultado estándar:

$$
\Gamma_{L M}=\frac{\sqrt{N}\left(\overline{L M}_{N T}-E\left(L M_{i}^{T}\right)\right)_{d}}{\sqrt{V\left(L M_{i}^{T}\right)}} \rightarrow N(0,1),
$$

donde $E\left(L M_{i}^{T}\right)$ y $V\left(L M_{i}^{T}\right)$ son los valores esperados y la varianza del estadístico $L M_{i}^{T}$, respectivamente, cuyos valores simulados se pueden encontrar en Im, Lee y Tieslau (2005). Se debe notar que el estadístico de prueba LM se distribuye asintóticamente normalmente y no se ve afectado por la presencia de rupturas estructurales.

\section{c) La prueba de Carrion-i-Silvestre}

Carrion-i-Silvestre, Barrio-Castro y Lopez-Bazo (2005) desarrollaron una prueba para probar la hipótesis nula de estacionariedad en un conjunto de datos en panel que toma en cuenta múltiples rupturas estructurales, cuyo procedimiento se basa en la prueba univariada de Kwiatkowski, Phillips, Smichdt y Shin (KPSs) (Kwiatkowski et al., 1992). A continuación, describiremos brevemente el modelo de Carrion-i-Silvestre (CIS), diseñado para probar la hipótesis nula de estacionariedad que permite múltiples cambios estructurales en el panel de datos.

$$
y_{i t}=\alpha_{i}+\sum_{k=1}^{m_{i}} \phi_{i k} D U_{i, k, t}+\beta_{i} t+\sum_{k=1}^{m_{i}} \eta_{i, k} D T_{i, k, t}^{*}+\varepsilon_{i, t},
$$

donde $y_{i, t}$ se refiere a la variable $y$ para la unidad $i$ en el tiempo $t ; D U_{i, k, t}$ y $D T_{i, k, t}^{*}$ son variables dummy que se definen como $D U_{i, k, t}=1$ para $t>T_{b, k}^{i}$ y 0 de otra forma, y $D T_{i, k, t}^{*}=t-T_{b, k}^{i}$ para $t>T_{b, k}^{i}$ y 0 de otra forma; $T_{b, k}^{i}$ denota la $k$-ésima fecha de la ruptura para el $i$-ésimo individuo y $k=\left\{1, \ldots, m_{i}\right\}, m_{i} \geq 0$. La ecuación (10) tiene tres importantes características. Primero, permite rupturas estructurales en cada serie temporal individual, las cuales son capturadas por $\phi_{i, k}$ y $\eta_{i, k}$. En segundo lugar, las rupturas estructurales no están restringidas y, por ende, pueden ocurrir en diferentes puntos. Tercero, los individuos pueden tener un número diferente de rupturas estructurales, esto es $m_{i} \neq m_{j}$.

La prueba de la hipótesis nula de que la series en el panel son estacionarias se lleva a cabo siguiendo la propuesta de Hadri (2000), quien diseñó un estadístico de prueba que es simplemente la media de las pruebas de estacionariedad univariada en KPSS. El estadístico de prueba es de la forma: 


$$
\operatorname{LM}(\widehat{\lambda})=N^{-1} \sum_{i=1}^{N}\left(\widehat{\psi}_{i}^{-2} T^{-2} \sum_{t=1}^{T} \widehat{S}_{i, t}^{2}\right),
$$

donde $L M_{i}\left(\hat{\lambda}_{i}\right)=\widehat{\psi}_{i}^{-2} T^{-2} \sum_{t=1}^{T} \widehat{S}_{i, t}^{2}$ es la prueba univariada KPSS para la unidad $i, \mathrm{y}$ $\widehat{S}_{i, t}=\sum_{j=1}^{t} \widehat{\varepsilon}_{i, j}$ se mantiene para el proceso de sumas parciales que se obtiene usando los residuos del método de mínimos ordinarios cuadrados (MCO) de la ecuación (10), donde $\psi_{i}^{2}$ es el estimador consistente de la varianza de los residuos, $\varepsilon_{i, t}$, de largo plazo. Esto permite que las perturbaciones sean heterocedásticas entre la dimensión de las unidades de sección cruzada. Aun más, se puede asumir homogeneidad reemplazando $\psi_{i}^{2}$ en la ecuación (11) con $\psi_{i}^{2}=N^{-1} \sum_{i=1}^{N} \widehat{\psi}_{i}^{2}$.

El estadístico de prueba para la hipótesis nula de un panel estacionario con múltiples rupturas es:

$$
Z(\widehat{\lambda})=\frac{\sqrt{N}(L M(\widehat{\lambda})-\bar{\xi})}{\bar{\varsigma}} N(0,1),
$$

donde $\bar{\xi}$ y $\bar{\varsigma}$ son calculadas como promedios de las medias individuales y las varianzas de $L M_{i}\left(\widehat{\lambda}_{i}\right)$, respectivamente. El cálculo del estadístico $Z(\widehat{\lambda})$ requiere que las series individuales sean independientes entre las unidades de sección cruzada, además de ser normales asintóticamente.

En tanto que $\lambda$ representa la dependencia de la prueba respecto a las fechas de las rupturas. Para cada individuo $i$, esto se define como:

$$
\lambda_{i}=\left(\lambda_{i, 1}, \ldots, \lambda_{i, m_{i}}\right)^{i}=\left(T_{b, 1}^{i} / T, \ldots, T_{b, m_{i}}^{i} / T\right) .
$$

Carrion-i-Silvestre, Barrio-Castro y Lopez-Bazo (2005) recomiendan que el número de los cambios estructurales y su posición se determinen a través del procedimiento de Bai y Perron (1998, 2001), el cual calcula la minimización de la suma de residuos al cuadrado (SSR). En éste, el procedimiento y la elección de las fechas de las rupturas se basan en el argumento que minimiza la secuencia de sSR individuales $\left(T_{b, 1}^{i} / T, \ldots, T_{b, m_{i}}^{i} / T\right)$ como sigue:

$$
\left(\widehat{T}_{b, 1}^{i} / T, \ldots, \widehat{T}_{b, m_{i}}^{i} / T\right)=\arg \min \operatorname{SSR}\left(T_{b, 1}^{i} / T, \ldots, T_{b, m_{i}}^{i} / T\right) .
$$

Siguiendo a Bai y Perron (2001), el número de rupturas estructurales asociado con cada individuo es estimado empleando el criterio informativo de 
Schwarz. Carrion-i-Silvestre, Barrio-Castro y Lopez-Bazo (2005) sugieren que el número máximo de rupturas sea cinco. Este procedimiento se repite $N$ veces con el fin de obtener el número estimado de rupturas y su ubicación para cada individuo. Simulaciones de Monte Carlo indican que la prueba no experimenta problemas en la potencia y tamaño de la prueba en muestras finitas (Basher y Carrión-i-Silvestre, 2008).

\section{La prueba de cointegración en panel con rupturas estructurales de Westerlund y Edgerton}

Con el fin de probar cointegración en el panel considerado se emplea la prueba de cointegración en panel con rupturas estructurales desarrollada por Westerlund y Edgerton (2008). Estos autores proponen dos versiones de ella, que se derivan de la prueba LM de raíz unitaria, para la hipótesis nula de no cointegración. Ambas versiones permiten heterocedasticidad y errores autocorrelacionados, interceptos y tendencias específicos por individuo o unidad, dependencia de sección cruzada, y rupturas estructurales desconocidas tanto en el intercepto como en la pendiente de la regresión cointegrante, las cuales se pueden localizar en fechas diferentes para las distintas unidades (Lee, 2013). Ellos consideran la siguiente ecuación:

$$
y_{i, t}=\alpha_{i}+\eta_{i t} t+\delta_{i} D_{i t}+X_{i t}^{\prime} \beta_{i}+\left(D_{i t} X_{i t}\right) \gamma_{i}+z_{i t} \text {, }
$$

donde $x_{i t}=x_{i t-1}+w_{i t}$ es un vector de dimensión $k$ que contiene los regresores $\mathrm{y}$ sigue un proceso de caminata aleatoria pura, y $D_{i t}$ es un escalar para la variable dummy de la ruptura tal que $D_{i t}=1$ sí $t>T_{i}$ y 0 de otra forma.

La perturbación $z_{i t}$ se asume que sigue un proceso generador de datos que permite dependencia de sección cruzada y se expresa como sigue:

$$
\begin{gathered}
z_{i t}=\lambda^{\prime} F_{t}+v_{i t}, \\
\phi_{i}(L) \Delta v_{i t}=\phi_{i} v_{i t-1}+e_{i t},
\end{gathered}
$$

donde $F_{t}$ es un vector de dimensión $r$ de factores comunes no observables $F_{j t}=\rho_{j} F_{j t-1}+u_{j t}$ con $j=1,2, \ldots, r ; \phi_{i}(L):=1-\sum_{j=1}^{p_{i}} \phi_{i j} L^{j}$ es un polinomio escalar, y $\lambda_{i}$ es un vector conformable de parámetros. Si se asume que $\rho_{j}<1$ para todo $j, F_{t}$ es estrictamente estacionario. Así, la relación en la ecuación (15) está cointegrada si $\phi_{i}<0$ y es espuria si $\phi_{i}=0$.

Para un $N$ dado, en la medida que $T \rightarrow \infty$ entonces $N / T \rightarrow 0$; el valor asintótico normalizado del estadístico de prueba se define como sigue: 
64 ECONOMÍA: TEORÍA Y PRÁCTICA • Nueva Época, número 44, enero-junio 2016

$$
z_{j}(N)=\sqrt{N}\left[\overline{L M}_{j}(N)-E\left(B_{j}\right)\right] \rightarrow N\left[0, \operatorname{var}\left(B_{j}\right)\right], \quad j=\phi, \tau .
$$

Aquí, $\overline{L M_{j}}(N)$ es el promedio de $\overline{L M}_{j}(i)$, y $B_{j}$ es la integración de un proceso browniano estándar.

\section{El estimador de Pedroni}

El estimador de Pedroni (PDOLS) parte de considerar el siguiente modelo:

$$
y_{i, t}=\alpha_{i}+\beta_{i} x_{i, t}+\mu_{i, t} .
$$

Éste es una extensión del estimador individual de series temporales de mínimos cuadrados ordinarios dinámicos (DOLS), el cual, a pesar de ser eficiente, es un estimador individual de la ecuación cointegrante que se puede aplicar a datos no estacionarios (Neal, 2014). Pedroni $(1999,2004)$ lo extiende a datos en panel de series temporales a través de una regresión de DoLs para cada individuo en el panel anterior, como sigue:

$$
y_{i, t}=\alpha_{i}+\beta_{i} x_{i, t}+\sum_{j=-P}^{P} \gamma_{i, j} \Delta x_{i, t-j}+\mu_{i t}^{*}
$$

donde $i=1,2, \ldots, N$ es el número de unidades en el panel; $t=1,2, \ldots, T$, el número de periodos de tiempo; $p=1,2, \ldots, P$, el número de rezagos y adelantos en la regresión DOLS; $\beta_{i}$, el coeficiente de la pendiente, y $x_{i, t}$, la variable explicativa. Los coeficientes $\beta$ y sus estadísticos $t$ asociados se promedian sobre la totalidad del panel a través del método de la media del grupo de Pedroni (Neal, 2014).

$$
\begin{gathered}
\hat{\beta}_{G M}^{*}=\left[\frac{1}{n} \sum_{i=1}^{n}\left(\sum_{t=1}^{T} z_{i, t} z_{i, t}^{\prime}\right)^{-1}\left\{\sum_{t=1}^{T} z_{i, t}\left(y_{i, t}-\bar{y}_{i}\right)\right\}\right] \\
t_{\hat{\beta}_{i}^{*}}=\left(\widehat{\beta}_{i}^{*}-\beta_{0}\right)\left\{\widehat{\sigma}_{i}^{-2} \sum_{t=1}^{T}\left(x_{i, t}-\bar{x}_{i}\right)^{2}\right\}^{\frac{1}{2}} \\
t_{\hat{\beta}_{G M}^{*}}=\frac{1}{\sqrt{N}} \sum_{i=1}^{N} t_{\hat{\beta}_{i}^{*}},
\end{gathered}
$$


donde $z_{i, t}$ es el vector $2(p+1) \times 1$ de regresores, el cual incluye los rezagos y los adelantos de las diferencias de la variable explicativa, y $\sigma_{i}^{2}$ es la varianza de largo plazo de los residuos $\mu_{i t}^{*}$.

Por el contrario, Kao y Chiang (1997) y Mark y Sul (2003) calculan los estadísticos del panel a través de la dimensión within, con el estadístico $t$ diseñado para probar $\mathrm{H}_{0}: \beta_{i}=\beta_{0}$ contra $\mathrm{H}_{A}: \beta_{i}=\beta_{A} \neq \beta_{0}$. El estimador PDOLS esta promediado a través de la dimensión between, es decir, la media del grupo. De acuerdo con esto, la prueba del estadístico del panel es $\mathrm{H}_{0}: \beta_{i}=\beta_{0}$, contra $\mathrm{H}_{A}: \beta_{i} \neq \beta_{0}$. En la hipótesis alternativa, los regresores no están restringidos a ser una constante $\beta_{A}$. Pedroni (2001) argumenta que esto es una importante ventaja de los estimadores de panel de series temporales que se basan en la dimensión between, particularmente cuando se espera heterogeneidad en las pendientes.

\section{ANÁLISIS ECONOMÉTRICO}

El panel de datos contiene series temporales continuas, de 1951 a 2010, del PIB per cápita de un conjunto de 17 países de América Latina. En primer lugar, se somete a prueba la hipótesis de convergencia en su versión restricta con los países de América Latina con respecto al promedio de la región, $D_{1} y_{i t}$, en el periodo en cuestión. La grafica 1 muestra la evolución del diferencial del logaritmo natural del PIB per cápita de cada uno de los países que conforman el panel con respecto al promedio de la región.

Como se puede apreciar en las gráficas de $D_{1} y_{i t}$ para los países de la región, la mayoría de ellos presenta una ligera tendencia con algunos quiebres, pero en algunos casos -Chile, Colombia, Ecuador, El Salvador, Paraguay, Perú y Uruguay-, más que una obvia tendencia, el quiebre parece inducir un cambio de nivel en la serie en cuestión. Debido a que no es del todo claro si el comportamiento del diferencial del PIB per cápita con respecto al promedio de la región presenta o no una tendencia en el grupo de países considerados, se efectuaron las pruebas propuestas por Carrion-i-Silvestre, Barrio-Castro y Lopez-Bazo (2005) únicamente con efectos individuales en un caso y en el otro. Además de estos efectos, se incorporó una tendencia. Los resultados de las pruebas aplicadas a $D_{1} y_{i t}$ se presentan en los cuadros 1 y 2 .

El cuadro 3 muestra los resultados de las pruebas LM de raíz unitaria sin rupturas, con una ruptura y con dos rupturas aplicadas al diferencial del PIB per cápita de los países con respecto al promedio de la región en forma logarítmica. Como se desprende de dicho cuadro, las pruebas de raíces unitarias en panel sin 
66 ECONOMÍA: TEORÍA Y PRÁCTICA • Nueva Época, número 44, enero-junio 2016

\section{Gráfica 1. Evolución de ly ${ }_{\mathrm{it}}$ con respecto al promedio de los países de América Latina considerados, 1951-2010}
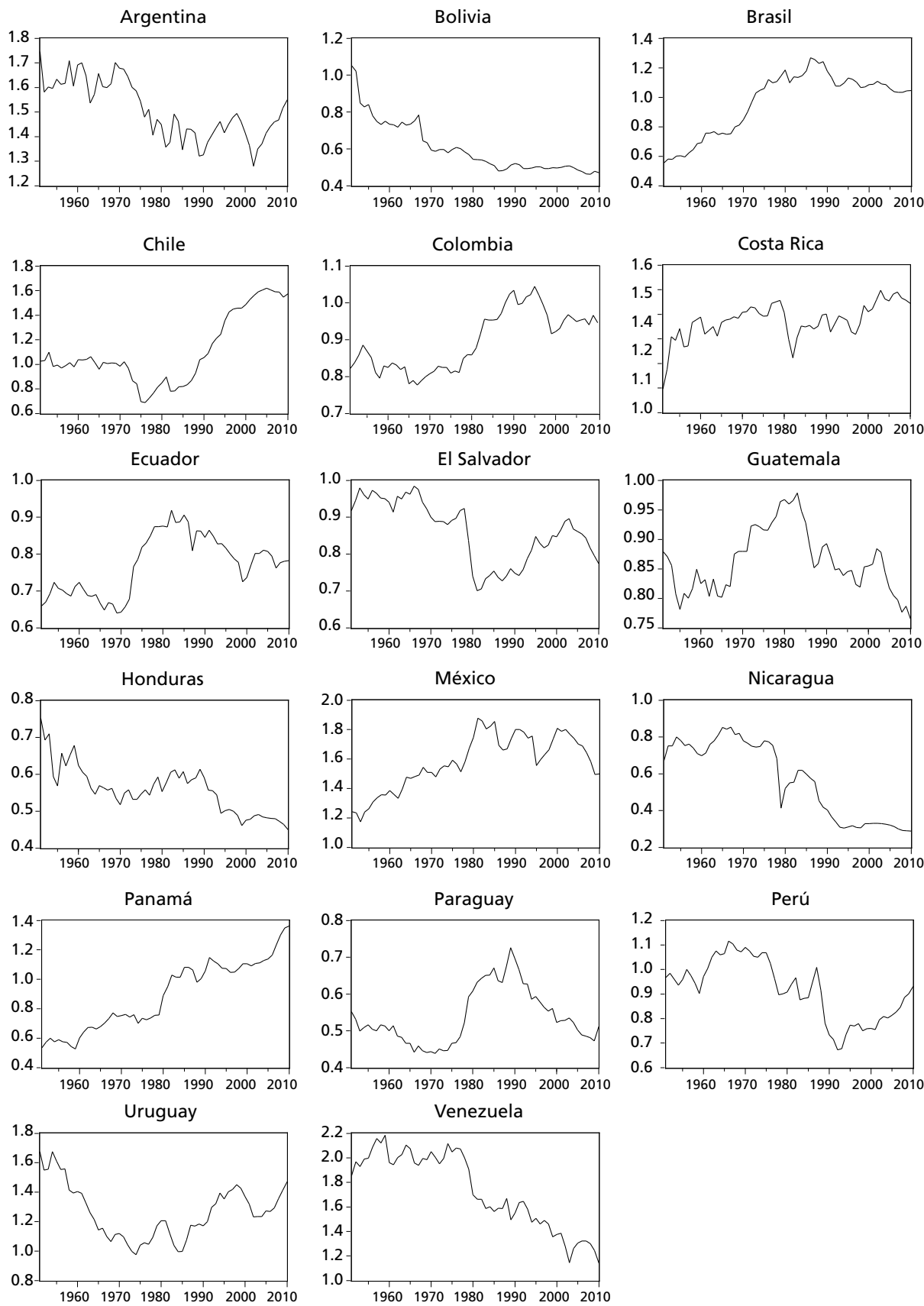
Cuadro 1. Estimación del número de rupturas estructurales para $\mathrm{D}_{1} \mathrm{y}_{\mathrm{it}}$ con respecto al promedio

Pruebas KPSS individuales
Con efectos individuales

Fuente: Elaboración propia.

Con efectos individuales y tendencia

\begin{tabular}{|c|c|c|c|c|c|c|}
\hline \multicolumn{7}{|c|}{ Pruebas KPSS individuales } \\
\hline & \multicolumn{3}{|c|}{ Con efectos individuales } & \multicolumn{3}{|c|}{ Con efectos individuales y tendencia } \\
\hline & Barlett & Cuadrático & $\begin{array}{l}\text { Fechas de } \\
\text { ruptura }\end{array}$ & Barlett & Cuadrático & $\begin{array}{c}\text { Fechas de } \\
\text { ruptura }\end{array}$ \\
\hline Argentina & 0.051 & 0.051 & 1975 & 0.054 & 0.054 & 1975,2001 \\
\hline Bolivia & 0.131 & 0.137 & 1967,1979 & 0.091 & 0.091 & 1959,1988 \\
\hline Brasil & 0.131 & 0.133 & 1959,1971 & 0.020 & 0.020 & 1971,1989 \\
\hline Chile & 0.043 & 0.047 & 1972, 1988, 1997 & 0.021 & 0.022 & $1974,1984,1996$ \\
\hline Colombia & 0.046 & 0.046 & 1981 & 0.088 & 0.088 & 1964, 1981, 1997 \\
\hline Costa Rica & 0.120 & 0.125 & 1959,1998 & 0.045 & 0.045 & 1960,1980 \\
\hline Ecuador & 0.045 & 0.048 & 1972,1994 & 0.036 & 0.036 & 1972, 1981, 2001 \\
\hline El Salvador & 0.032 & 0.032 & 1969, 1979, 1992 & 0.054 & 0.055 & 1979, 2001 \\
\hline Guatemala & 0.051 & 0.050 & 1971,1985 & 0.125 & 0.119 & $1967,1984,2000$ \\
\hline Honduras & 0.068 & 0.067 & 1960,1993 & 0.137 & 0.146 & 1970, 1990 \\
\hline México & 0.046 & 0.048 & 1963,1978 & 0.065 & 0.065 & 1980, 1999 \\
\hline Nicaragua & 0.045 & 0.049 & 1978,1989 & 0.039 & 0.039 & $1978,1987,1996$ \\
\hline Panamá & 0.127 & 0.127 & 1961, 1979, 2001 & 0.026 & 0.025 & 1980 \\
\hline Paraguay & 0.049 & 0.051 & 1961, 1978, 1996 & 0.028 & 0.028 & 1969, 1978, 1991 \\
\hline Perú & 0.026 & 0.027 & $\begin{array}{c}1961,1976,1988 \\
2001\end{array}$ & 0.031 & 0.031 & 1976,1988 \\
\hline Uruguay & 0.152 & 0.154 & 1962,1991 & 0.026 & 0.026 & $1973,1982,2000$ \\
\hline Venezuela & 0.215 & 0.215 & 1979,1998 & 0.032 & 0.034 & 1979 \\
\hline
\end{tabular}

rupturas, con y sin tendencia, muestran que cuando se ignoran las rupturas no es posible rechazar la hipótesis nula de la raíz unitaria en la prueba restricta de convergencia de los países de América Latina con respecto al promedio de la región al nivel de significación convencional. Por el contrario, cuando se realizan con una y con dos rupturas, las pruebas rechazan por mucho la hipótesis de la raíz unitaria para $D_{1} y_{i t}$, sugiriendo de este modo convergencia de los países de la región, tanto individualmente como en el panel en su conjunto, con respecto al PIB per cápita promedio de la región.

Por su parte, las pruebas de estacionariedad de Hadri (2000) con efectos individuales y sin rupturas aplicadas al panel en cuestión, tanto con el núcleo espectral de Barlett como con el cuadrático, independientemente de la suposición relativa a la heterogeneidad de la varianza estimada en el largo plazo, rechazan contundentemente la hipótesis nula de estacionariedad de $D_{1} y_{i t}$, incluso si se toman los valores críticos de la prueba obtenidos por bootstraping, los 
Cuadro 2. Pruebas de raíces unitarias en panel de CIS para $\mathrm{D}_{1} \mathrm{y}_{\mathrm{it}}$ con respecto al promedio

\begin{tabular}{|c|c|c|c|c|c|c|c|c|}
\hline \multicolumn{9}{|c|}{ Barlett } \\
\hline & \multicolumn{4}{|c|}{ Con efectos individuales } & \multicolumn{4}{|c|}{ Con efectos individuales y tendencia } \\
\hline & Estadístico & $\begin{array}{l}\text { Valor } \\
\text { de } p\end{array}$ & $10 \%$ & $5 \%$ & Estadístico & $\begin{array}{l}\text { Valor } \\
\text { de } p\end{array}$ & $10 \%$ & $5 \%$ \\
\hline $\begin{array}{l}\text { Sin rupturas } \\
\text { (homogéneo) }\end{array}$ & 35.044 & {$[0.000]$} & 3.715 & 5.125 & 8.339 & {$[0.000]$} & 4.024 & 5.186 \\
\hline $\begin{array}{l}\text { Sin rupturas } \\
\text { (heterogéneo) }\end{array}$ & 30.106 & {$[0.000]$} & 3.345 & 4.729 & 7.147 & {$[0.000]$} & 3.776 & 4.772 \\
\hline $\begin{array}{l}\text { Con rupturas } \\
\text { (homogéneo) }\end{array}$ & 0.827 & {$[0.204]$} & 7.219 & 7.886 & 4.259 & {$[0.000]$} & 13.275 & 14.089 \\
\hline $\begin{array}{l}\text { Con rupturas } \\
\text { (heterogéneo) }\end{array}$ & 1.968 & {$[0.025]$} & 6.522 & 7.160 & 12.862 & {$[0.000]$} & 16.110 & 16.948 \\
\hline $\begin{array}{l}\text { Sin rupturas } \\
\text { (homogéneo) }\end{array}$ & 35.984 & {$[0.000]$} & 3.908 & 5.472 & 8.682 & {$[0.000]$} & 3.900 & 5.118 \\
\hline $\begin{array}{l}\text { Sin rupturas } \\
\text { (heterogéneo) }\end{array}$ & 30.634 & {$[0.000]$} & 3.381 & 4.930 & 7.286 & {$[0.000]$} & 3.957 & 4.953 \\
\hline $\begin{array}{l}\text { Con rupturas } \\
\text { (homogéneo) }\end{array}$ & 1.090 & {$[0.138]$} & 7.217 & 8.045 & 4.393 & {$[0.000]$} & 12.446 & 13.213 \\
\hline $\begin{array}{l}\text { Con rupturas } \\
\text { (heterogéneo) }\end{array}$ & 2.128 & {$[0.017]$} & 6.447 & 7.056 & 13.022 & {$[0.000]$} & 15.468 & 16.405 \\
\hline
\end{tabular}

Fuente: Elaboración propia.

cuales se calculan en el caso de que las unidades del panel presenten dependencia de sección cruzada. Un resultado similar presenta la prueba de Hadri (2000) cuando ésta se especifica con efectos individuales y tendencia. De esta manera, no señala indicios de convergencia de los países de la región con respecto a su promedio.

A su vez, los resultados de las pruebas de Carrion-i-Silvestre, BarrioCastro y Lopez-Bazo (2005) con rupturas aplicadas a $D_{1} y_{i t}$ con respecto al promedio de la región no permiten rechazar la hipótesis nula de estacionariedad, ya sea que se asuma dependencia o independencia en las unidades de sección cruzada en el panel en presencia de una varianza homogénea. Si, por el contrario, se considera una varianza heterogénea, se rechaza la hipótesis nula de estacionariedad en el panel asumiendo independencia, pero no es posible rechazarla en el caso de dependencia de las unidades. Cuando esta prueba con rupturas se especifica con efectos individuales y tendencia, se rechaza la hipótesis nula de estacionariedad, asumiendo independencia, en tanto que no es posible rechazar esta hipótesis cuando se asume dependencia de las unidades. Teniendo en cuenta es- 
Cuadro 3. Prueba $L M$ de raíz unitaria sin rupturas, con una ruptura $y$ con dos rupturas, para $\mathrm{D}_{1} \mathrm{y}_{\mathrm{it}}$ con respecto al promedio

\begin{tabular}{|c|c|c|c|c|c|c|}
\hline & \multicolumn{2}{|c|}{ Sin rupturas } & \multicolumn{2}{|c|}{ Con una ruptura } & \multicolumn{2}{|c|}{ Con dos rupturas } \\
\hline & $\begin{array}{c}\text { Sin } \\
\text { tendencia }\end{array}$ & $\begin{array}{c}\text { Con } \\
\text { tendencia }\end{array}$ & & & & \\
\hline & Estadístico $p$ & Estadístico $p$ & Estadístico $p$ & Ruptura & Estadístico $p$ & Rupturas \\
\hline Argentina & -1.279 & $-1.337(8)$ & -3.702 (7) & 1983 & -5.105 & 1969,1983 \\
\hline Bolivia & $-1.793(0)$ & $-1.669(0)$ & $-3.344 \quad(0)$ & 1990 & -5.318 & 1961,1987 \\
\hline Brasil & $-1.294(7)$ & -1.238 (7) & $-3.582(7)$ & 1986 & -5.904 & 1966,1979 \\
\hline Chile & $-1.580(7)$ & $-1.636(7)$ & -2.923 & 1985 & -5.017 & 1973,1992 \\
\hline Colombia & -1.578 & -1.679 (5) & -3.210 & 1979 & -4.492 & 1976,1996 \\
\hline Costa Rica & $-1.940(0)$ & $-1.852(0)$ & $-3.589(0)$ & 1980 & -4.812 & 1963,1981 \\
\hline Ecuador & $-1.464(0)$ & -1.908 & $-3.345(6)$ & 1981 & -4.460 & 1971,1993 \\
\hline El Salvador & $-2.023(6)$ & -2.300 & $-3.114 \quad(1)$ & 1985 & $-4.696 \quad(6)$ & 1977,1996 \\
\hline Guatemala & $-1.325(0)$ & $-1.393(0)$ & -3.215 (7) & 1975 & -4.438 & 1965,1974 \\
\hline Honduras & -1.858 & -1.334 (4) & -3.269 (3) & 1970 & -5.088 & 1969,1985 \\
\hline México & $-1.597(0)$ & $-1.770(0)$ & $-4.953 \quad(2)$ & 1977 & -6.399 & 1984,2001 \\
\hline Nicaragua & $-1.654(0)$ & $-1.633(0)$ & $-3.488 \quad(3)$ & 1977 & -6.536 & 1978,1991 \\
\hline Panamá & $-2.904(8)$ & $-2.895(8)$ & $-3.725(8)$ & 1992 & -5.328 & 1978,1994 \\
\hline Paraguay & -1.913 & $-1.876 \quad(4)$ & $-2.767 \quad(6)$ & 1976 & -5.169 & 1976,1989 \\
\hline Perú & $-1.444 \quad(2)$ & -1.862 & -3.810 & 1986 & -6.630 & 1964,1989 \\
\hline Uruguay & -1.310 & -1.288 & $-4.359 \quad(6)$ & 1976 & $-4.826 \quad(6)$ & 1969,1992 \\
\hline Venezuela & $-2.348 \quad(0)$ & $-2.439 \quad(0)$ & $-3.853 \quad(0)$ & 1980 & $-5.983 \quad(7)$ & 1978,1988 \\
\hline Panel & 1.380 & 1.084 & $-11.169 \quad(0)$ & 1980 & $-23.134 \quad(7)$ & 1978,1988 \\
\hline
\end{tabular}

Nota: Los números entre paréntesis son los rezagos incorporados en cada prueba. Los valores críticos correspondientes a los niveles de significación de $1 \%, 5 \%$ y $10 \%$ para el estadístico panel LM sin rupturas, con una y con dos rupturas son $-2.32,-1.64$ y -1.28 , respectivamente.

Fuente: Elaboración propia.

tos resultados, las pruebas de raíces unitarias en panel de Carrion-i-Silvestre, Barrio-Castro y Lopez-Bazo (2005) con rupturas estructurales, ya sea que se especifiquen con o sin tendencia, sugieren estacionariedad en $l y_{t}$ con respecto al promedio de la región, bajo el supuesto de dependencia de sección cruzada y, de esta forma, convergencia de los países de la región con respecto a este indicador.

En segundo lugar, se procedió a probar la hipótesis de convergencia de los países de América Latina con respecto a EU, $D_{1} y_{i t}$, a través de la prueba restricta en el periodo en cuestión. La gráfica 2 muestra la evolución del diferencial del logaritmo natural del PIB per cápita de cada uno de los países que conforman el panel con respecto al PIB per cápita estadounidense.

Al igual que en el caso del diferencial con respecto al PIB promedio de la región, no queda del todo claro si la especificación correcta de la prueba debe incorporar una tendencia o únicamente se registra un cambio de nivel en la tota- 
70 ECONOMÍA: TEORÍA Y PRÁCTICA • Nueva Época, número 44, enero-junio 2016

\section{Gráfica 2. Evolución de ly $_{\mathrm{it}}$ con respecto a EU de los países de América Latina considerados, 1951-2010}
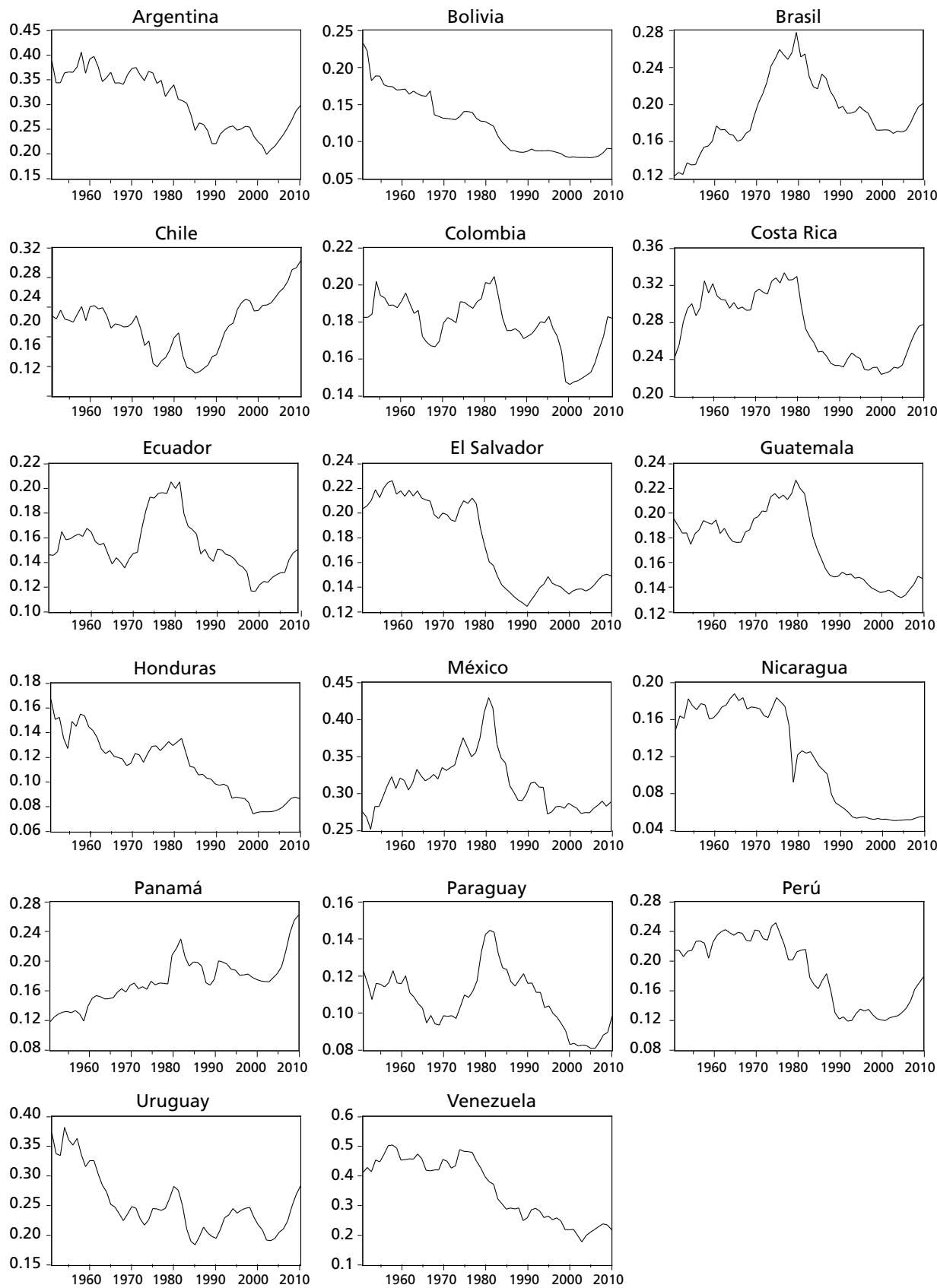
lidad de los individuos del panel en cuestión. Por tal razón, los cuadros 4 y 5 muestran los resultados de las pruebas de Hadri (2000) y de Carrion-i-Silvestre, Barrio-Castro y Lopez-Bazo (2005) para ambas especificaciones.

Los resultados de las pruebas LM de raíz unitaria sin rupturas, con una ruptura y con dos rupturas aplicadas al diferencial del PIB per cápita de los países con respecto a EU en forma logarítmica se presentan en el cuadro 6. Como se puede ver en dicho cuadro, no es posible rechazar la hipótesis nula de raíz unitaria en el panel cuando la prueba se especifica sin rupturas; por el contrario, la prueba se rechaza en forma contundente cuanto se incorporan una y dos ruptu-

$$
\begin{aligned}
& \text { Cuadro 4. Estimación del número de rupturas estructurales para ly }{ }_{i t} \\
& \text { con respecto a EU }
\end{aligned}
$$

Pruebas KPSS individuales

Con efectos individuales

\begin{tabular}{lcccccc} 
& Barlett & Cuadrático & $\begin{array}{c}\text { Fechas de } \\
\text { ruptura }\end{array}$ & Barlett & Cuadrático & $\begin{array}{c}\text { Fechas de } \\
\text { ruptura }\end{array}$ \\
\hline Argentina & 0.031 & 0.031 & 1975,1984 & 0.033 & 0.033 & $1977,1990,2001$ \\
Bolivia & 0.159 & 0.152 & 1967,1983 & 0.028 & 0.028 & 1983 \\
Brasil & 0.051 & 0.051 & $1959,1970,88$ & 0.056 & 0.056 & $1965,1976,2001$ \\
Chile & 0.029 & 0.031 & $1972,1991,2001$ & 0.024 & 0.024 & $1972,1982,1998$ \\
Colombia & 0.095 & 0.095 & 1997 & 0.049 & 0.049 & $1964,1983,1998$ \\
Costa Rica & 0.097 & 0.102 & 1982 & 0.049 & 0.049 & $1960,1980,1991,2001$ \\
Ecuador & 0.047 & 0.045 & $1973,1983,1995$ & 0.024 & 0.024 & $1961,1972,1982,1998$ \\
El Salvador & 0.036 & 0.039 & 1967,1980 & 0.031 & 0.031 & 1979,1990 \\
Guatemala & 0.036 & 0.038 & $1971,1984,1996$ & 0.076 & 0.076 & $1964,1979,1988,2001$ \\
Honduras & 0.044 & 0.046 & $1962,1983,1993$ & 0.139 & 0.151 & $1968,1982,1998$ \\
México & 0.126 & 0.126 & $1959,1973,1985,1994$ & 0.118 & 0.125 & 1979,1988 \\
Nicaragua & 0.037 & 0.039 & $1978,1987,1996$ & 0.092 & 0.092 & $1978,1987,1996$ \\
Panamá & 0.011 & 0.012 & 1960,1979 & 0.060 & 0.060 & $1979,1990,2001$ \\
Paraguay & 0.034 & 0.036 & $1963,1977,1986,1996$ & 0.020 & 0.021 & $1965,1978,1999$ \\
Perú & 0.018 & 0.017 & 1977,1988 & 0.073 & 0.073 & $1976,1988,2001$ \\
Uruguay & 0.075 & 0.075 & $1962,1982,1991$ & 0.049 & 0.049 & $1973,1982,2000$ \\
Venezuela & 0.054 & 0.060 & 1982,1998 & 0.018 & 0.018 & $1959,1973,1990,2001$ \\
\hline
\end{tabular}


ras, sugiriendo de esta manera que el panel conformado por el diferencial del PIB per cápita, con respecto al de EU, de los países de América Latina es estacionario y que, por tanto, en este caso existe convergencia de los países de la región a la luz de la prueba con cambios de nivel.

Cuadro 5. Pruebas de raíces unitarias en panel CIS para $\mathrm{l}_{\text {it }}$ con respecto a EU (Ho: estacionariedad del panel)

\begin{tabular}{|c|c|c|c|c|}
\hline \multicolumn{5}{|c|}{ Con efectos individuales } \\
\hline & \multicolumn{2}{|c|}{ Barlett } & \multicolumn{2}{|c|}{$\begin{array}{l}\text { Valores críticos por } \\
\text { bootstraping }\end{array}$} \\
\hline & Estadístico & Valor de $p$ & $10 \%$ & $5 \%$ \\
\hline Sin rupturas (homogéneo) & 43.674 & [0.000] & 4.157 & 6.634 \\
\hline Sin rupturas (heterogéneo) & 42.311 & {$[0.000]$} & 3.808 & 5.536 \\
\hline Con rupturas (homogéneo) & -2.777 & [0.997] & 8.538 & 9.012 \\
\hline \multirow[t]{3}{*}{ Con rupturas (heterogéneo) } & 0.050 & [0.480] & 7.909 & 8.504 \\
\hline & \multicolumn{2}{|c|}{ Cuadrático } & \multicolumn{2}{|c|}{$\begin{array}{c}\text { Valores críticos por } \\
\text { bootstraping }\end{array}$} \\
\hline & Estadístico & Valor de $p$ & $10 \%$ & $5 \%$ \\
\hline Sin rupturas (homogéneo) & 44.761 & {$[0.000]$} & 4.266 & 6.009 \\
\hline Sin rupturas (heterogéneo) & 42.617 & {$[0.000]$} & 3.662 & 5.227 \\
\hline Con rupturas (homogéneo) & -2.695 & [0.996] & 8.464 & 8.943 \\
\hline Con rupturas (heterogéneo) & 0.142 & [0.444] & 7.950 & 8.540 \\
\hline \multicolumn{5}{|c|}{ Con efectos individuales y tendencia } \\
\hline & \multicolumn{2}{|c|}{ Barlett } & \multicolumn{2}{|c|}{$\begin{array}{l}\text { Valores críticos por } \\
\text { bootstraping }\end{array}$} \\
\hline & Estadístico & Valor de $p$ & $10 \%$ & $5 \%$ \\
\hline Sin rupturas (homogéneo) & 4.772 & {$[0.000]$} & 4.145 & 5.428 \\
\hline Sin rupturas (heterogéneo) & 6.376 & {$[0.000]$} & 4.363 & 5.618 \\
\hline Con rupturas (homogéneo) & 9.622 & {$[0.000]$} & 11.640 & 13.320 \\
\hline \multirow[t]{3}{*}{ Con rupturas (heterogéneo) } & 17.955 & {$[0.000]$} & 25.588 & 28.367 \\
\hline & \multicolumn{2}{|c|}{ Cuadrático } & \multicolumn{2}{|c|}{$\begin{array}{l}\text { Valores críticos por } \\
\text { bootstraping }\end{array}$} \\
\hline & Estadístico & Valor de $p$ & $10 \%$ & $5 \%$ \\
\hline Sin rupturas (homogéneo) & 4.842 & {$[0.000]$} & 4.005 & 5.304 \\
\hline Sin rupturas (heterogéneo) & 6.389 & {$[0.000]$} & 4.197 & 5.367 \\
\hline Con rupturas (homogéneo) & 9.772 & {$[0.000]$} & 11.648 & 13.060 \\
\hline Con rupturas (heterogéneo) & 18.570 & {$[0.000]$} & 24.873 & 26.922 \\
\hline
\end{tabular}

Fuente: Elaboración propia. 
Cuadro 6. Prueba LM de raíz unitaria sin rupturas, con una ruptura $y$ con dos rupturas, para $l y_{i t}$ con respecto a $E U$

\begin{tabular}{lccccccc}
\hline \multicolumn{3}{c}{ Sin rupturas } & \multicolumn{2}{c}{ Con una ruptura } & \multicolumn{2}{l}{ Con dos rupturas } \\
\hline & Sin tendencia & Con tendencia & & & & \\
\hline & Estadístico $p$ & Estadístico $p$ & Estadístico $p$ & Ruptura & Estadístico $p$ & Rupturas \\
Argentina & $-1.015(8)$ & $-1.337(8)$ & $-3.702(7)$ & 1983 & $-5.105(7)$ & 1969,1983 \\
Bolivia & $-1.948(0)$ & $-1.669(0)$ & $-3.344(0)$ & 1990 & $-5.318(6)$ & 1961,1987 \\
Brasil & $-1.186(8)$ & $-1.238(7)$ & $-3.542(7)$ & 1986 & $-5.904(7)$ & 1966,1979 \\
Chile & $-1.418(1)$ & $-1.636(7)$ & $-2.923(7)$ & 1985 & $-5.017(3)$ & 1973,1992 \\
Colombia & $-2.109(1)$ & $-1.679(5)$ & $-3.210(8)$ & 1979 & $-4.492(5)$ & 1976,1996 \\
Costa Rica & $-1.933(5)$ & $-1.852(0)$ & $-3.589(0)$ & 1980 & $-4.812(1)$ & 1963,1981 \\
Ecuador & $-2.367(3)$ & $-1.908(3)$ & $-3.345(6)$ & 1981 & $-4.460(6)$ & 1971,1993 \\
El Salvador & $-1.489(5)$ & $-2.300(1)$ & $-3.114(1)$ & 1985 & $-4.696(6)$ & 1977,1996 \\
Guatemala & $-1.948(5)$ & $-1.393(0)$ & $-3.215(7)$ & 1975 & $-4.438(7)$ & 1965,1974 \\
Honduras & $-2.380(3)$ & $-1.334(4)$ & $-3.269(3)$ & 1970 & $-5.088(3)$ & 1969,1985 \\
México & $-1.411(6)$ & $-1.770(0)$ & $-4.953(2)$ & 1977 & $-6.399(3)$ & 1984,2001 \\
Nicaragua & $-1.239(0)$ & $-1.633(0)$ & $-3.488(3)$ & 1977 & $-6.536(8)$ & 1978,1991 \\
Panamá & $-1.961(5)$ & $-2.895(8)$ & $-3.725(8)$ & 1992 & $-5.328(8)$ & 1978,1994 \\
Paraguay & $-2.049(2)$ & $-1.876(4)$ & $-2.767(6)$ & 1976 & $-5.169(4)$ & 1976,1989 \\
Perú & $-1.486(1)$ & $-1.862(1)$ & $-3.810(8)$ & 1986 & $-6.630(1)$ & 1964,1989 \\
Uruguay & $-2.027(8)$ & $-1.288(1)$ & $-4.359(6)$ & 1976 & $-4.826(6)$ & 1969,1992 \\
Venezuela & $-0.790(8)$ & $-2.439(0)$ & $-3.853(0)$ & 1980 & $-5.983(7)$ & 1978,1988 \\
\hline Panel & 1.493 & 1.084 & $-11.169(0)$ & 1980 & $-23.134(7)$ & 1978,1988 \\
\hline
\end{tabular}

Notas: Los números entre paréntesis son los rezagos incorporados en cada prueba. Los valores críticos correspondientes a los niveles de significación de 1\%,5\% y 10\% para el estadístico panel LM sin rupturas, con una y dos rupturas son - 2.32, - 1.64 y - 1.28, respectivamente.

Fuente: Elaboración propia.

A su vez, las pruebas de estacionariedad de Hadri (2000) sin rupturas, con efectos individuales y sin tendencia, tanto con el núcleo espectral de Barlett como con el cuadrático referente a la suposición relativa a la heterogeneidad de la varianza estimada en el largo plazo, presentadas en el cuadro 4, rechazan conjuntamente la hipótesis nula de estacionariedad de las series del panel en cuestión. Este resultado se mantiene incluso cuando se relaja este supuesto de independencia de las unidades de sección cruzada cuando la prueba se especifica con efectos individuales únicamente. Por el contrario, no es posible rechazar la hipótesis nula 
de estacionariedad en el panel cuando se relaja el supuesto de independencia entre las unidades del panel, incorporando efectos individuales y tendencia en la especificación, al nivel de significación de $5 \%$ para el caso homogéneo.

De igual forma, los resultados de la prueba de Carrion-i-Silvestre, Barrio-Castro y Lopez-Bazo (2005) con rupturas aplicadas a la versión restricta se muestran en el cuadro 5. Cuando la prueba se especifica con efectos individuales en ningún caso, con y sin independencia de las unidades de sección cruzada, se

Cuadro 7. Resultados de las pruebas de cointegración en panel con y sin rupturas estructurales de Westerlund y Edgerton entre el PIB per cápita de países de Latinoamérica y el de EU

\begin{tabular}{|c|c|c|c|c|c|c|}
\hline & \multicolumn{3}{|c|}{$k=1$} & \multicolumn{3}{|c|}{$k=2$} \\
\hline & Sin cambio & $\begin{array}{c}\text { Cambio de } \\
\text { nivel }\end{array}$ & $\begin{array}{l}\text { Cambio de } \\
\text { régimen }\end{array}$ & Sin cambio & $\begin{array}{c}\text { Cambio de } \\
\text { nivel }\end{array}$ & $\begin{array}{c}\text { Cambio de } \\
\text { régimen }\end{array}$ \\
\hline & -0.831 & -0.002 & 0.645 & 0.826 & 0.396 & 1.621 \\
\hline$Z_{\tau}(N)$ & [0.203] & [0.499] & [0.741] & [0.796] & [0.654] & [0.948] \\
\hline \multirow[t]{3}{*}{$Z_{\phi}(N)$} & 0.106 & 0.556 & 1.255 & 1.245 & 1.034 & 1.986 \\
\hline & [0.542] & [0.711] & [0.895] & [0.893] & [0.849] & [0.977] \\
\hline & \multicolumn{3}{|c|}{ Rupturas } & & \multicolumn{2}{|c|}{ Rupturas } \\
\hline Argentina & & 1988 & 2001 & & 1988 & 2001 \\
\hline Bolivia & & 1967 & 1967 & & 1967 & 1967 \\
\hline Brasil & & 1980 & 1980 & & 1980 & 1980 \\
\hline Chile & & 1981 & 1974 & & 1981 & 1974 \\
\hline Colombia & & 1998 & 1998 & & 1998 & 1998 \\
\hline Costa Rica & & 1980 & 1981 & & 1980 & 1981 \\
\hline Ecuador & & 1972 & 1972 & & 1972 & 1972 \\
\hline El Salvador & & 1978 & 1978 & & 1978 & 1978 \\
\hline Guatemala & & 1967 & 1982 & & 1967 & 1982 \\
\hline Honduras & & 1998 & 1998 & & 1998 & 1998 \\
\hline México & & 1994 & 1994 & & 1994 & 1994 \\
\hline Nicaragua & & 1978 & 1978 & & 1978 & 1978 \\
\hline Panamá & & 1979 & 1979 & & 1979 & 1979 \\
\hline Paraguay & & 1978 & 1978 & & 1978 & 1978 \\
\hline Perú & & 1988 & 1988 & & 1988 & 1988 \\
\hline Uruguay & & 1981 & 1981 & & 1981 & 1981 \\
\hline Venezuela & & 1988 & 2002 & & 1988 & 2002 \\
\hline
\end{tabular}

Nota: $k$ es el número de factores comunes incorporados en la prueba. Fuente: Elaboración propia. 
rechaza la hipótesis nula de estacionariedad del panel. Mientras que, por el contrario, no se rechaza únicamente bajo el supuesto de dependencia en el panel. De esta manera, encontramos evidencia de convergencia de los países de la región con respecto a EU con la prueba de estacionariedad del panel en presencia de rupturas estructurales.

Con el fin de reforzar o refutar esta evidencia, se verifica ahora la posibilidad de que las variables estudiadas se encuentren cointegradas. Los cuadros $7 \mathrm{y}$ 8 muestran los resultados de las pruebas de cointegración en panel con rupturas

Cuadro 8. Resultados de las pruebas de cointegración en panel con y sin rupturas estructurales de Westerlund y Edgerton entre el PIB per cápita de países de Latinoamérica y el promedio de la región

\begin{tabular}{|c|c|c|c|c|c|c|}
\hline & \multicolumn{3}{|c|}{$k=1$} & \multicolumn{3}{|c|}{$k=2$} \\
\hline & Sin cambio & $\begin{array}{c}\text { Cambio de } \\
\text { nivel }\end{array}$ & $\begin{array}{l}\text { Cambio de } \\
\text { régimen }\end{array}$ & Sin cambio & $\begin{array}{c}\text { Cambio de } \\
\text { nivel }\end{array}$ & $\begin{array}{l}\text { Cambio de } \\
\text { régimen }\end{array}$ \\
\hline & 0.274 & 0.885 & -0.243 & 1.545 & 0.435 & -0.090 \\
\hline \multirow[t]{2}{*}{$Z_{\tau}(N)$} & {$[0.608]$} & [0.812] & [0.404] & [0.939] & [0.668] & [0.464] \\
\hline & 0.790 & 1.268 & 0.661 & 1.753 & 1.031 & 0.676 \\
\hline \multirow[t]{2}{*}{$Z_{\phi}(N)$} & {$[0.785]$} & [0.898] & {$[0.746]$} & [0.960] & [0.849] & {$[0.751]$} \\
\hline & \multicolumn{3}{|c|}{ Rupturas } & & \multicolumn{2}{|c|}{ Rupturas } \\
\hline Argentina & & 1988 & 1988 & & 1988 & 1988 \\
\hline Bolivia & & 1967 & 1967 & & 1967 & 1967 \\
\hline Brasil & & 1980 & 1980 & & 1980 & 1980 \\
\hline Chile & & 1981 & 1981 & & 1981 & 1981 \\
\hline Colombia & & 1998 & 1998 & & 1998 & 1998 \\
\hline Costa Rica & & 1981 & 1981 & & 1981 & 1981 \\
\hline Ecuador & & 1972 & 1972 & & 1972 & 1972 \\
\hline El Salvador & & 1979 & 1979 & & 1979 & 1979 \\
\hline Guatemala & & 1967 & 1967 & & 1967 & 1967 \\
\hline Honduras & & 1993 & 1964 & & 1993 & 1964 \\
\hline México & & 1994 & 1994 & & 1994 & 1994 \\
\hline Nicaragua & & 1978 & 1978 & & 1978 & 1978 \\
\hline Panamá & & 1979 & 1979 & & 1979 & 1979 \\
\hline Paraguay & & 1978 & 1978 & & 1978 & 1978 \\
\hline Perú & & 1988 & 1988 & & 1988 & 1988 \\
\hline Uruguay & & 1981 & 1981 & & 1981 & 1981 \\
\hline Venezuela & & 1988 & 1988 & & 1988 & 1988 \\
\hline
\end{tabular}

Nota: $k$ es el número de factores comunes incorporados en la prueba. Fuente: Elaboración propia. 
de Westerlund y Edgerton (2008) entre el PIB per cápita de los países de América Latina considerados en la muestra y el de EU, en el primero, y con respecto al promedio de la región, en el segundo.

Como se puede apreciar, cuando la prueba se efectúa con uno y dos factores comunes, en ningún caso se puede rechazar la hipótesis nula de no cointegración en el panel bajo cualquier especificación: sin cambios, con un cambio en la media y con un cambio en tendencia. Estos resultados no refuerzan la evidencia anterior sobre convergencia absoluta arrojada por la versión restricta de las pruebas de raíces unitarias con rupturas.

De manera análoga a los resultados de las pruebas de raíces unitarias en panel con rupturas, las pruebas de cointegración en panel muestran que los países presentan fechas de rupturas heterogéneas. Por último, con fines de inspección, el cuadro 9 presenta los resultados de las estimaciones de la pendiente de la versión irrestricta de la prueba con el estimador PDOLs en el supuesto de cointegración en panel entre el PIB per cápita de los países de la región tanto con respecto a su promedio como al de EU. ${ }^{1}$

En relación con este último, los únicos países para los cuales no fue posible rechazar la hipótesis nula de que la pendiente de la ecuación (2) es igual a la unidad y, por ende, de convergencia hacia esta economía de referencia fueron Costa Rica, El Salvador y Paraguay. En cambio, con respecto al promedio, los países para los cuales no es posible rechazar la hipótesis nula de que el parámetro es igual a la unidad son Chile, Brasil y Honduras. De esta forma, estos resultados tampoco refuerzan la evidencia de convergencia absoluta mostrada por las pruebas de raíces unitarias y estacionariedad en panel con rupturas y, más bien, ponen de manifiesto que no hay evidencia de convergencia absoluta y posiblemente evidencia de convergencia condicional. Tales resultados están acordes a lo encontrado por Rodríguez, Perrotini y Venegas-Martínez (2012) y Rodríguez, Mendoza y Perrotini (2015).

\section{CONCLUSIONES}

La cuestión de si grupos de países o de regiones convergen hacia algún referente de economía líder ha acaparado la atención de muchos investigadores en tiempos

\footnotetext{
${ }^{1} \mathrm{~A}$ diferencia del estimador para paneles cointegrados que se usa en este trabajo, Rodríguez, Perrotini y Venegas-Martínez (2012) emplean los estimadores de Mark y Sul (2003) y de Kao y Chiang (2000), mientras que Rodríguez, Perrotini y Mendoza (2014) utilizan el estimador del grupo de medias (mean group, $\mathrm{MG}$ ) y el estimador de medias agrupadas (pooled mean group, $\mathrm{PMG}$ ).
} 
Cuadro 9. Estimaciones de la pendiente de cointegración individuales y de panel entre el PIB per cápita de países de Latinoamérica con respecto al de EU y al promedio de la región mediante PDOLS

\begin{tabular}{|c|c|c|c|c|}
\hline & \multicolumn{2}{|c|}{ Con respecto a EU } & \multicolumn{2}{|c|}{ Con respecto al promedio } \\
\hline & Coeficiente & Estadístico $t$ & Coeficiente & Estadístico $t$ \\
\hline Argentina & 0.681 & $-8.867^{* \star *}$ & 0.471 & $-11.700^{* \star *}$ \\
\hline Bolivia & 0.232 & $-18.040^{* * *}$ & 0.148 & $-21.940^{* * *}$ \\
\hline Brasil & 1.642 & $7.663^{* \star *}$ & 1.171 & $1.891^{*}$ \\
\hline Chile & 1.393 & $2.936^{\star \star *}$ & 1.060 & 0.711 \\
\hline Colombia & 1.139 & $4.048^{* * *}$ & 0.836 & $-5.324^{* * *}$ \\
\hline Costa Rica & 0.998 & -0.089 & 0.705 & $-6.786^{* * *}$ \\
\hline República Dominicana & 1.137 & $2.472^{* * *}$ & 0.805 & $-3.020^{* * *}$ \\
\hline Ecuador & 0.673 & $-7.715^{* * *}$ & 0.473 & $-10.460^{* \star *}$ \\
\hline El Salvador & 0.936 & -1.329 & 0.651 & $-5.987^{* \star *}$ \\
\hline Guatemala & 0.594 & $-10.200^{\star * *}$ & 0.416 & $-13.480^{\star \star *}$ \\
\hline Honduras & 1.254 & $6.201^{* * *}$ & 0.902 & $-1.781^{*}$ \\
\hline México & -0.410 & $-8.920^{* * *}$ & -0.377 & $-13.000^{* \star *}$ \\
\hline Nicaragua & 1.839 & $15.420^{\star \star *}$ & 1.354 & $7.087^{* * *}$ \\
\hline Paraguay & 1.066 & 0.990 & 0.789 & $-3.113^{* * *}$ \\
\hline Perú & 0.554 & $-5.484^{\star \star *}$ & 0.356 & $-8.557^{* * *}$ \\
\hline Uruguay & 0.799 & $-2.372^{* *}$ & 0.597 & $-7.500^{* * *}$ \\
\hline Venezuela & 0.297 & $-9.845^{* * *}$ & 0.168 & $-13.050^{* * *}$ \\
\hline Panel & 0.872 & $-8.035^{* * *}$ & 0.619 & $-28.130^{* * *}$ \\
\hline
\end{tabular}

Nota: ${ }^{* *}, * * *$ indican el rechazo de la hipótesis nula $H_{0}: \beta_{i}=1$, a niveles de significación de $10 \%, 5 \%$ y $1 \%$, respectivamente.

Fuente: Elaboración propia.

recientes. Con el fin de dilucidarlo, se han aplicado diversas técnicas estadísticas y econométricas para confirmar o descartar el cumplimiento de la hipótesis de convergencia.

En este trabajo se han empleado diversas pruebas, tanto de raíz unitaria como de estacionariedad y de cointegración en panel, con y sin rupturas estructurales, para constatar la hipótesis de convergencia en una muestra de países de América Latina con respecto a dos referentes: el promedio de la región y la economía de EU, considerada como líder. Esto se hizo mediante la prueba restricta de convergencia, que consiste en averiguar si el diferencial del PIB per cápita de 
los países considerados con respecto a la economía de referencia, en forma logarítmica, es estacionario.

Las pruebas de raíces unitarias, tanto con cambios de nivel, como proponen Im, Lee y Tieslau (2005), con una y dos rupturas, como las de estacionariedad de Carrion-i-Silvestre, Barrio-Castro y Lopez-Bazo (2005), y la posibilidad de incluir efectos fijos con y sin tendencia, sugieren convergencia de los países de América Latina en relación con el PIB per cápita estadounidense y con el promedio, con ambas pruebas en presencia de rupturas estructurales. Cabe añadir que la prueba de Carrion-i-Silvestre es mucho más flexible, ya que no sólo permite tomar en cuenta la heterogeneidad, sino también otra serie de características posiblemente presentes en el panel, como 1) múltiples rupturas estructurales, 2) rupturas determinadas endógenamente para fechas desconocidas y 3) un diferente número de rupturas para cada individuo.

En contraposición con los anteriores resultados, los de las pruebas de cointegración en panel con rupturas estructurales de Westerlund y Edgerton (2008) entre el PIB per cápita de los países de América Latina considerados y el de EU muestran que en ningún caso fue posible rechazar la hipótesis nula de no cointegración en el panel bajo cualquier especificación: sin cambios, con un cambio en media y con un cambio en tendencia. Estos resultados no refuerzan la evidencia previa sobre la hipótesis de convergencia absoluta arrojada por la versión restricta con las pruebas de raíces unitarias con rupturas.

Finalmente, los resultados de las estimaciones de la pendiente de la versión irrestricta de la prueba con el estimador PDOLs, bajo el supuesto de cointegración en panel entre el PIB per cápita de los países de la región tanto con respecto a su promedio como con respecto al de EU, muestran que en muy pocos casos no fue posible rechazar la hipótesis nula de que la pendiente de la ecuación en la prueba irrestricta es igual a la unidad y, por ende, de convergencia hacia estos referentes. Las únicas excepciones fueron Costa Rica, El Salvador y Paraguay, con respecto a EU, y Chile, Brasil y Honduras, con respecto al PIB promedio de la región.

De esta manera, las pruebas de raíces unitarias con rupturas estructurales no brindan evidencia concluyente sobre la existencia de convergencia absoluta en la región, en virtud de que sus resultados no son corroborados por las pruebas de cointegración con rupturas estructurales ni por los estimadores para paneles cointegrados, como el que se empleó en este trabajo. En todo caso, pueden estar reflejando procesos de convergencia condicional, como lo reportan otros trabajos empíricos sobre el tema. 


\section{REFERENCIAS BIBLIOGRÁFICAS}

Álvarez, Inmaculada; Lucas, Sonia de, y Delgado, María de Jesús (2009), “Globalización y convergencia económica: un análisis empírico para los países de América Latina", Paradigma Económico, 1 (1), pp. 34-53.

Amable, Bruno, y Juillard, Michel (2000), The Historical Process of Convergence. [PDF] Consultado el 15 de enero de 2016, en: http://www.jourdan.ens.fr/ amable/convergence.pdf

Amsler, Christine, y Lee, Junsoo (1995), "An LM test for unit root in the presence of a structural change", Econometric Theory, 11, pp. 359-368.

Bai, Jushan, y Perron, Pierre (1998), "Estimating and testing linear models with multiple structural changes", Econometrica, 66 (1), pp. 47-78.

- (2001), "Multiple Structural Change Models: A Simulation Analysis", reporte técnico.

Barro, Robert, y Sala-i Martin, Xavier (2004), Economic Growth, 2a. ed., Cambridge, The MIT Press.

Basher, Syed A., y Carrion-i-Silvestre, Josep Lluis (2008), "Deconstructing shocks and persistence in OECD real exchange rates", Xarxa de Referència en Economia Aplicada Working Papers XrEAP2008-6.

Bernard, Andrew, y Durlauf, Steven (1995), "Convergence in International Output", Journal of Applied Econometrics, 10 (2), pp. 97-108.

_ (1996), "Interpreting tests of the convergence hypothesis", Journal of Econometrics, 71 (1-2), pp. 161-173.

Beyaert, Arielle, y Camacho, Máximo (2008), "TAR Panel Unit Root Tests and Real Convergence", Review of Development Economics, 3 (12), pp. 668-681.

Camarero, Mariam; Flores, Renato, y Tamarit, Cecilio (2002), "Multivariate time series evidence of international output convergence in Mercosur", Computing in Economics and Finance, (0) 87, s.p.

Carrion-i-Silvestre, Josep Lluis; Barrio-Castro, Tomás del, y Lopez-Bazo, Enrique (2005), "Breaking the panels: an application to the GDP per capita", Econometrics Journal, 8 (2), pp. 159-175.

Carrion-i-Silvestre, Josep Lluis, y German-Soto, Vicente (2007), "Stochastic Convergence amongst Mexican States”, Regional Studies, 41 (4), pp. 531-541

Cermeño, Rodolfo, y Llamosas, Irving (2007), "Convergencia del PIB por habitante de 6 países emergentes con Estados Unidos: un análisis de cointegración”, EconoQuantum, 4 (1), pp. 59-84. 
Cheung, Yin-Wong, y Garcia, Antonio (2004), "Testing for output convergence: a reexamination", Oxford Economic Papers, 56 (1), pp. 45-63.

Díaz-Pedroza, Jesús; Sánchez-Vargas, Armando, y Mendoza-González, Miguel Ángel (2009), "Convergencia hacia la economía regional líder en México. Un análisis de cointegración en panel”, El Trimestre Económico, 76 (2), pp. 407-431.

Durlauf, Steven (2000), "Econometric Analysis and the Study of Economic Growth: a Skeptical Perspective, Working Papers 10, Wisconsin MadisonSocial Systems.

Easterly, William; Fiess, Nobert, y Lederman, Daniel (2003), "NAFTA and Convergence in North America: High Expectations, Big Events, Little Time", LACEA Economía, 4 (1), pp. 1-53.

Evans, Paul (1997), "How fast do economies converge?", Review of Economics and Statistics, 79 (2), pp. 219-225.

Fajnzylber, Fernando (1990), Industrialización en América Latina: de la “caja negra” al “casillero vacio”, Cuadernos de la CEPAL 60, Santiago de Chile, CEPAL.

Gregory, Allan W., y Hansen, Bruce E. (1996), "Residual-Based Tests for Cointegration in Models with Regime Shifts", Journal of Econometrics, 70 (1), pp. 99-126.

Hadri, Kaddour (2000), "Testing for Stationarity in Heterogeneous Panel Data”, Econometrics Journal, 3 (2), 148-161.

Im, Kyung-So; Lee, Junsoo, y Tieslau, Margie (2005), "Panel LM unit Root Tests with Level Shifts", Oxford Bulletin of Economics and Statistics, 67 (3), 393-419.

Kao, Chihwa, y Chiang, Min-Hsien (2000), "On the Estimation and Inference of a Cointegrated Regression in Panel Data", en B. Baltagi, T. B. Fomby y R. Carter Hill (eds.), Nonstationary Panels, Panel Cointegration, and Dynamic Panels, Advances in Econometrics 15, s.1., Emerald, pp. 161-178.

Kwiatkowski, Denis; Phillips, Peter; Schmidt, Peter, y and Shin, Yongcheol (1992), "Testing the null hypothesis of stationarity against the alternative of a unit root: how sure are we that economic time series have a unit root?", Journal of Econometrics, 54 (1-3), pp.159-178.

Lee, Chien-Chiang (2013), "Insurance and real output: The key role of banking activities", Macroeconomic Dynamics, 17 (2), pp. 235-260.

Levin, Andrew, y Lin, Chien-Fu (1992), "Unit root tests in panel data: asymptotic and finite-sample properties", University of California, San Diego, documento de trabajo 92-93.

(1993): "Unit Root Tests in Panel Data: New Results", Department of Economics, UC-San Diego, documento de trabajo.

Levin, Andrew; Lin, Chien-Fu, y Chu, Chia-Sang (2002), "Unit root test in panel data: 
asymptotic and finite sample properties”, Journal of Econometrics, 108 (1), pp. 1-24.

Linden, Mikael (2000), "Testing growth convergence with time series data - a non-parametric approach", International Review of Applied Economics, 14 (3), pp. 361-70.

Mankiw, N. Gregory; Romer, David, y Weil, David N. (1992), "A contribution to the empirics of economic growth", Quarterly Journal of Economics, 107 (2), pp. 407-437.

Martín, Fernando (2010), “Convergencia en América Latina. Un análisis dinámico”, Instituto de Iberoamérica, Universidad de Salamanca, documento de trabajo DT 02/2010.

Mendoza, Jorge E. (2007), “Apertura, gasto público y convergencia en América Latina: un análisis econométrico espacial”, Comercio Exterior, 57 (9), pp. 705-717.

Mark, Nelson C., y Sul, Donggyu (2003), "Cointegration Vector Estimation by Panel DoLs and Long-run Money Demand", Oxford Bulletin of Economics and Statistics, 65 (5), pp. 655-680.

Neal, Timothy (2014), "Panel cointegration analysis with xtpedroni”, Stata Journal, 14 (3), 684-692.

Pedroni, Peter (1999), “Critical Values for Cointegration Tests in Heterogeneous Panels with Multiple Regressors", Oxford Bulletin of Economics and Statistics, 61 (especial), pp. 653-670.

_ (2001), "Purchasing power parity tests in cointegrated panels", Review of Economics and Statistics, 83 (4), pp. 727-731.

- (2004), "Panel Cointegration: Asymptotic And Finite Sample Properties Of Pooled Time Series Tests With An Application To The PPP Hypothesis", Econometric Theory, 20 (3), pp. 597-625.

Phillips, Peter, y Moon, Hyungsik (2000), "Nonstationary panel data analysis: an overview of some recent developments", Econometric Reviews, 19 (3), pp. 263-286.

Quah, Danny T. (1993), “Galton's Fallacy and the Convergence Hypothesis”, Scandinavian Journal of Economics, 95 (4), pp. 427-443.

(1996), "Convergence Empirics across Economies with (some) Capital Mobility", Journal of Economic Growth, 1 (1), pp. 95-124.

Rodríguez, Domingo; López-Herrera, Francisco, y Venegas-Martínez, Francisco (2014), "Are there Economic Convergence Clubs in Latin America?", Journal of Economics and Development Studies, 2 (3), pp. 113-123.

Rodríguez, Domingo; Mendoza, Miguel Ángel, y Perrotini, Ignacio (2015), “Análisis no-lineal de la convergencia regional en América Latina, 1950-2010: un modelo panel TAR", Problemas del Desarrollo, 46 (182), pp. 119-142. 
82 ECONOMÍA: TEORÍA Y PRÁCTICA • Nueva Época, número 44, enero-junio 2016

Rodríguez, Domingo; Perrotini, Ignacio, y Mendoza, Miguel Ángel (2014), “Crecimiento económico y convergencia en América Latina, 1950-2010”, Monetaria, 36 (2), pp. 273-307.

Rodríguez, Domingo; Perrotini, Ignacio, y Venegas-Martínez, Francisco (2012), "La hipótesis de convergencia en América Latina: un análisis de cointegración en panel”, EconoQuantum, 9 (2), pp. 99-102.

Sanguinetti, Pablo, y Villar, Leonardo (2012), "Patrones de desarrollo en América Latina: ¿convergencia o caída en la trampa del ingreso medio?”, Coyuntura Económica: Investigación Económica y Social, 42 (2), pp. 155-188.

Schmidt, Peter, y Phillips, Peter (1992), "LM Tests for a Unit Root in the Presence of Deterministic Trends", Oxford Bulletin of Economics and Statistics, 54 (3), pp. 257-287.

Westerlund, Joakim, y Edgerton, David (2008), "A simple test for cointegration in dependent panels with structural breaks", Oxford Bulletin of Economics and Statistics, 70 (5), pp. 665-704.

Zheng, Bingwen (2011), “The 'Middle Income Trap' and China's path to development: international experiences and lessons", China Economist, 6 (3), pp. 4-16. 